\title{
Cloud Computing and Accident Handling Systems
}

\author{
Jabar $\mathrm{H}$ Yousif \\ Faculty of Computing and IT, Sohar University, \\ Oman
}

\author{
Dinesh Kumar Saini \\ Faculty of Computing and IT, Sohar University, \\ Oman \\ Faculty of Engineering and IT, University of \\ Queensland, Australia
}

\begin{abstract}
An attempt is made to study the current issues of the cloud computing solutions for the life critical system- car accident systems in the Gulf region. Gulf region has high death rate because of car accidents and there is little or no proper accident handling facilities in the region. This Research paper includes the review of development in the field of cloud computing in the service industries which provide some assistance to the accident handling systems including the hospitals and the drivers using latest technologies such as Mobile computing, SaaS, Cloud Computing etc..
\end{abstract}

\section{General Terms}

Software Systems, Computing, Cloud, Accident Handling Systems, Life Critical Systems

\section{Keywords}

SaaS, Cloud computing, Systems

\section{INTRODUCTION}

Car is a life critical system and operating engine need technical expertise, if the system is not performing or driver make any mistake it lead to accident. An implementation such life critical systems results in large changes in the way the legacy systems works. Transport vehicles which includes like bus, car, trucks and other two wheelers are life critical because they are used by the human beings. The vehicles are growing exponential and the advancement in the vehicles is also growing [1].

The role of information technology in averting accidents and curbing the number of road accidents will be studied through the analysis of data.

The Police employ some of the following IT Systems to control road safety, like Fare and passenger control systems, Road Electronic Monitors, Display screens for public transport, intelligent traffic control systems, Passenger traffic control systems, Traffic video monitoring systems, Navigation systems, and Signal control systems [2].

These systems generate tera bytes of data that need to be analyzed, not only to produces statistics but also to generate solutions to curb disastrous accidents. The analysis of this data will provide solutions to make road safe and also throw light on the ways to avert deaths and plight of victims in the aftermath of an accident [4]. This data is rarely studied.

Analysis of these data can shed light on how to discover, hot routes in road networks, relation between driver attention (and fatigue) and road accident through Fatality Analysis Reporting System, Effect of weather on the frequency and fatality of road accidents, Identify, target and reduce occupational road safety risks [3].

The research paper is aimed to study the existing systems for accidents and will implement artificial intelligent techniques and data mining techniques for analyzing the data [5]. The data is collected from the ROP for the year 2009-2010 and the data shows the numbers and factors.

\section{Traffic Statistics}

\section{$2009=2010$}

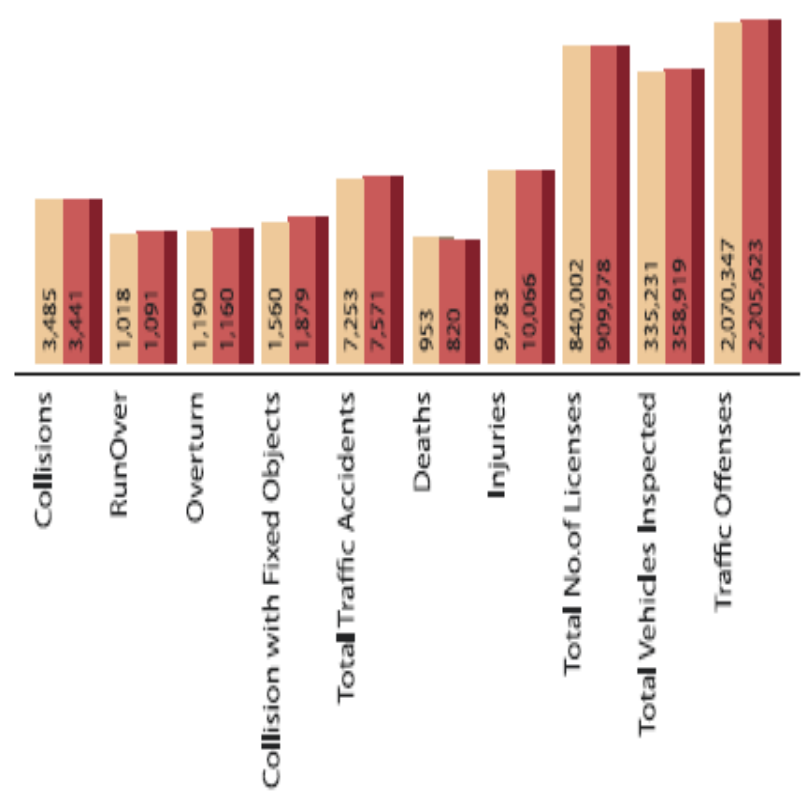

Figure1: Traffic Statistics

\section{RELATED WORK}

In today's time most of the vehicles are having equipment like smart phone devices, sensors with more powerful sensing, communication and processing capabilities devices. AV-track system is suggested using mobile phone in order to improve energy efficiency and sensor reliability [6].

Various soft computing techniques like use hidden Markov model based mapping scheme and Viterbi decoding scheme to estimate travel time and the simulation results validate the performance of their V-Track system. This kind of vehicle systems use soft computing but there is lack of global computing issues.

The authors in [7] emphasized a cooperative transit tracking method with the aid of built-in sensors inside the smart phone which provide better control over the vehicle. An accelerometer based activity recognition algorithm is proposed to decide user riding behavior and another method for tracking underground vehicles is also given.

The author [8] presented novel navigation architecture for vehicles with comprehensive explanation of all sub-layers. Intermediate system enables the functions such as perception, localization, mapping, real time traffic and tracking etc. A smart car sensor network platform based on the OSGi 
framework and AMI-C standards. They proposed an Architecture named SCSN with detailed hardware design and implementation.

In order to monitor road traffic the authors in [9] propose a novel traffic control system based on their new design methodology and WSN.

The provision of services in real time for mobile vehicles is always a challenge research. Various researchers proposed development of a sensor system for mobile cyclist named as net depends on the wireless sensor network. Equipped with different types of sensors on the bike to organize bicycle area network (BAN) and can be anyone in the network data exchange in real time by updating common stock.

A mobile vehicle system named ParkNet is designed and implement in [19]. In order to get good accuracy, they used GPS device and ultrasonic rangefinder, real time parking statistics. The authors present a review of using 4th generation (4G) wireless technology in cars and it is concepts. A practical application platform is provided to demonstrate particular attractive services in the upcoming time.

In [18] authors presented a survey for platform-as-a Service (PaaS) public cloud requirements with the some applications in an interactive environment, such as a car.

In [22] the authors proposed a cloud computing support technology for comparison between existing on-demand bus system and the innovative on-demand bus system. They simulated the cloud computing bus system based on developing new algorithm.

The concept of vehicular cloud computing is presented in [16]. The researchers focused on untapped resource sharing in the car such as Internet connection computing, storage, etc.

\section{CLOUD COMPUTING IN THE ACCIDENT HANDLING}

Cloud computing in car accidents handling is an emerging paradigm, fast and timely computing resources such as processing, communication and storage are needed while handling the accident. There are many service providers which provides services over cloud so the services required for handling accidents can be designed in the accident cloud. For example, Amazon Web Services lets traffic police store traffic data via its Simple Storage Service (S3) and perform computations on traffic data using the Elastic Compute Cloud (EC2)[9].

This type of computing provides many advantages for accident handling. Reports suggest that while handling accident lot of computing resources are needed, the cloud for the life critical system is an emerging area. When the accident happened lot of quick and fast actions are needed for handling the injured and providing trauma care.

Faster communication services are needed and Cloud computing seems to offer some incredible benefits for communicators: the availability of an incredible array of software applications, access to lightning-quick processing power, unlimited storage, and the ability to easily share and process information [10]. All of this is available through browser any time you can access the Internet. "Could Computing" brings such a change-computer storage computing center are set up by professional network companies such as Google and IBM, through one cable user can access easily with browser, make "Could" as the center of material storage and application services $[11,12]$.

Police and hospitals can takes advantage of the use of mobile devices and wireless communication for data transmission related to accidents, so there is no need to install large and complex systems [13].

Third generation mobile systems such as UMTS (Universal Mobile Telecommunications System), will be set up in the future not far in one nation, revolutionized telecommunications technology by offering mobile users content rich services, wireless broadband access to Internet, and worldwide roaming [14].

\section{INFRASTRUCTURE MODELS FOR CLOUD COMPUTING SOLUTION}

The Police and hospitals who handle accident can decide the type of the boundaries of cloud computing which deploy their applications according to organization demands and objectives. Police and insurance companies are interested in the actual factor and the person responsible for the accident while the hospital and doctors are interested in the timely help and attainment of the injured people who is injured during accident in the trauma center. The cloud computing is classified in various categories especially it is divided into three main categories which are Public cloud, the private cloud and the hybrid as depicted in figure $2[15,16]$.

For accident and traffic related issues it is recommended to have on public cloud. The location is not imposed still it is advised to run the accident cloud on public category, public clouds are naturally "out of organization" on public sites on the Internet. Even special trauma care and post-accident handling issues can be hosted on private clouds which are positioned on premises of hospitals or police station or even the insurance companies.

Public clouds are very much transparent because the public clouds are run outside of organization by third parties which are not directly involved in any direct business, which force transparency in the system. Various applications of different agencies like police, hospitals and insurance providers can be put together on the cloud's servers, on one storage systems, and networks so that access to these services can be made more simpler, easier and faster.

Public clouds are very much needed in the developing countries because the expertise and trauma handling can be made simpler for these countries also. Traffic and accident cloud services very much needed mainly for traffic police and accident controllers, they present a way to diminish the accident risk and cost by providing a flexible, even momentary extension to cyber infrastructure needed by various agencies like police and hospitals [25].

Special Private Clouds can be constructed for the limited use of specific client like insurance companies, which can provide the utmost control over the data, security, and quality of service for the vehicle insurance or life insurance providers. The interested insurance companies has particular infrastructure and has a control over how their applications are organized on it for insurance claims and finding the right facts about the accidents. The Private clouds can be deployed inside the insurance companies' datacenter, and they also may be deployed at a collocation facility, like insurance companies have specific requirement then the police and government.

Hybrid clouds provide both public and private cloud types facilities. Hybrid cloud facilitates on-demand, externally 
provisioned scale facilities. Hybrid clouds are helpful to supplement a private cloud with the resources of a public cloud; workload and other kind of fluctuations can be minimized in the hybrid cloud. Most of the hybrid clouds support almost all web2.0 applications. Hybrid cloud can be very useful for traffic police and hospital trauma center unit service center; the hybrid cloud can be utilized to perform periodic tasks that can be deployed simply on a public cloud.

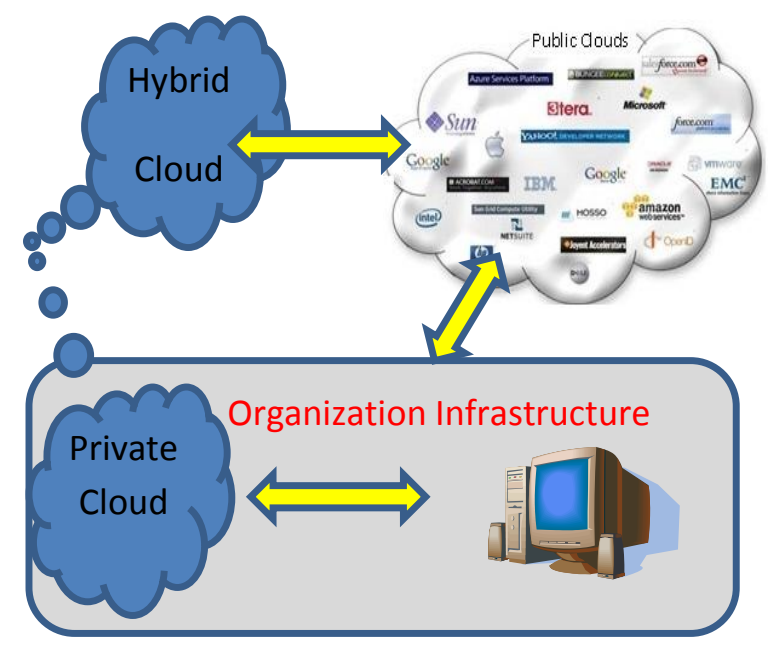

Figure2. Types of cloud computing

Cloud computing can illustrate the services being supplied at any of the established layers from hardware to applications. The police and insurance companies are interested in the vehicle and the person who is driving while hospital need for both. The cloud computing company provides a services that belongs to the SPI model of cloud computing. Typically, the cloud service providers like to offer services that can be collected into three categories:

a) Software as a service (SaaS)

SaaS as can be used for running the applications like HISHL-7 on demand while dealing with insurance client or even the insurance claims. The services of multiple channels like police, hospital and insurance are used a single version of the software that runs on the cloud.

b) Platform as a service (PaaS)

HIS-HL-7 can be used over the PaaS, a service covering a layer of software and presents it as a service that can be used to construct higher-level services for trauma centers or hospitals and insurance companies. PaaS can be visualized as either creator of the service or client of the service. PaaS can use various operating systems. Middle ware and application software running in the various hospitals, police control rooms and insurance companies. PaaS is API application and it provides encapsulated service to the various clients. c) Infrastructure as a service (IaaS)

Insurance companies and traffic police requires IaaS as service utilizes services such the storage. IaaS determine capabilities as modulated services over the network for various services need by various agencies like Police and Insurance companies. IaaS provides high-performance computing applications like Servers, storage systems, switches, routers.

Hardware as services (Haas) can also provide the outsource facility for maintaining servers, storage and networking equipment. Police, insurance companies and hospitals can use the facilities provided by the HaaS cloud service.

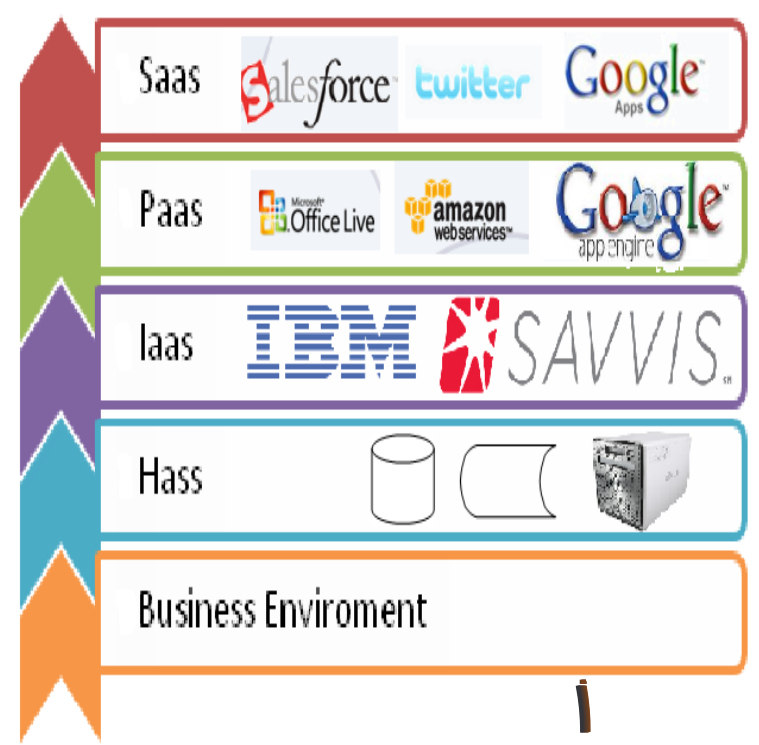

Figure 3 depicts the Architectural layers of cloud computing

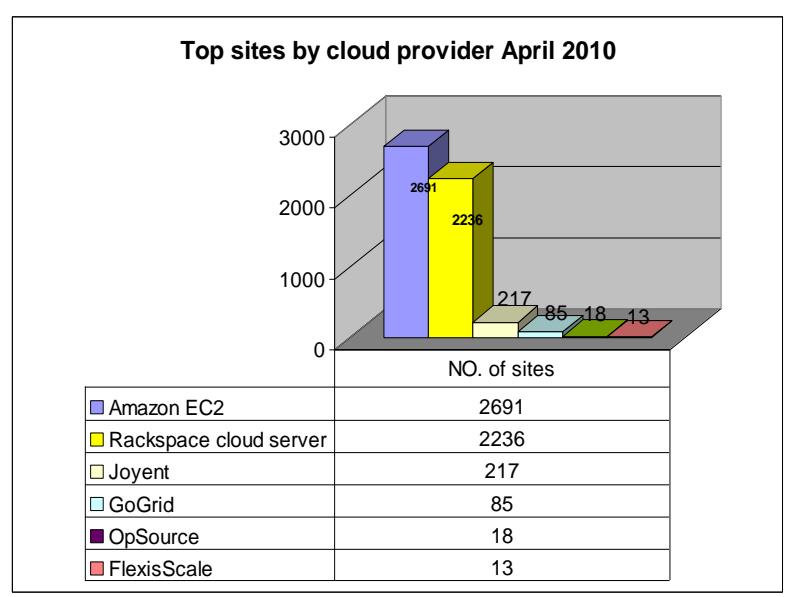

Figure4: The usage of cloud provider 
The above table shows the top companies who are offering various services to various hospital and accident handling. Special software architecture design and infrastructure requirements are there for proper handling of accidents. Cloud solutions which are especially designed for companies like insurance, police and hospitals will be very helpful in providing the required services.

In sake of facilitating the interaction between vehicle drivers and outside car world we proposed in this paper a vehicle cloud architecture based modern technologies such as wireless communication techniques, Internet and cloud computing etc. This can presents a future transportation system which will be more powerful, intellectual, flexible and suitable for mutually drivers and administrative centers. A number of customized and innovative cloud vehicle services like driver healthcare monitoring and in-car resource sharing services etc. By integrating the cloud computing technique, we intend to provide users a cheap real time services that follows and manages the driver information and situation.

\section{PROPOSED SYSTEM}

The proposed system has four main levels, which are:

1- Car level: it is suggested to use the tools that help to detect the problem and send the information to the nearest information center. The GPS, smart phone, sensor are used to gather the information like speed, gas level, driver behavior, etc. which is implemented in the Haas layer of the cloud.

2- Transmission \& communication level: this level is used to support the system with tools and technologies that used as a medium to send and receive the information across the layers.

3- Car Management Information \& Deployment (PaaS): Most of the processing jobs related to the data about car, driver, weather, location, health care companies, hospitals and insurance companies are analyzed and processed in this phase. This level is used to conclude the context information and implement the data analysis and mining phase for processing, and reasoning the valuable information to support the monitoring of decision making.

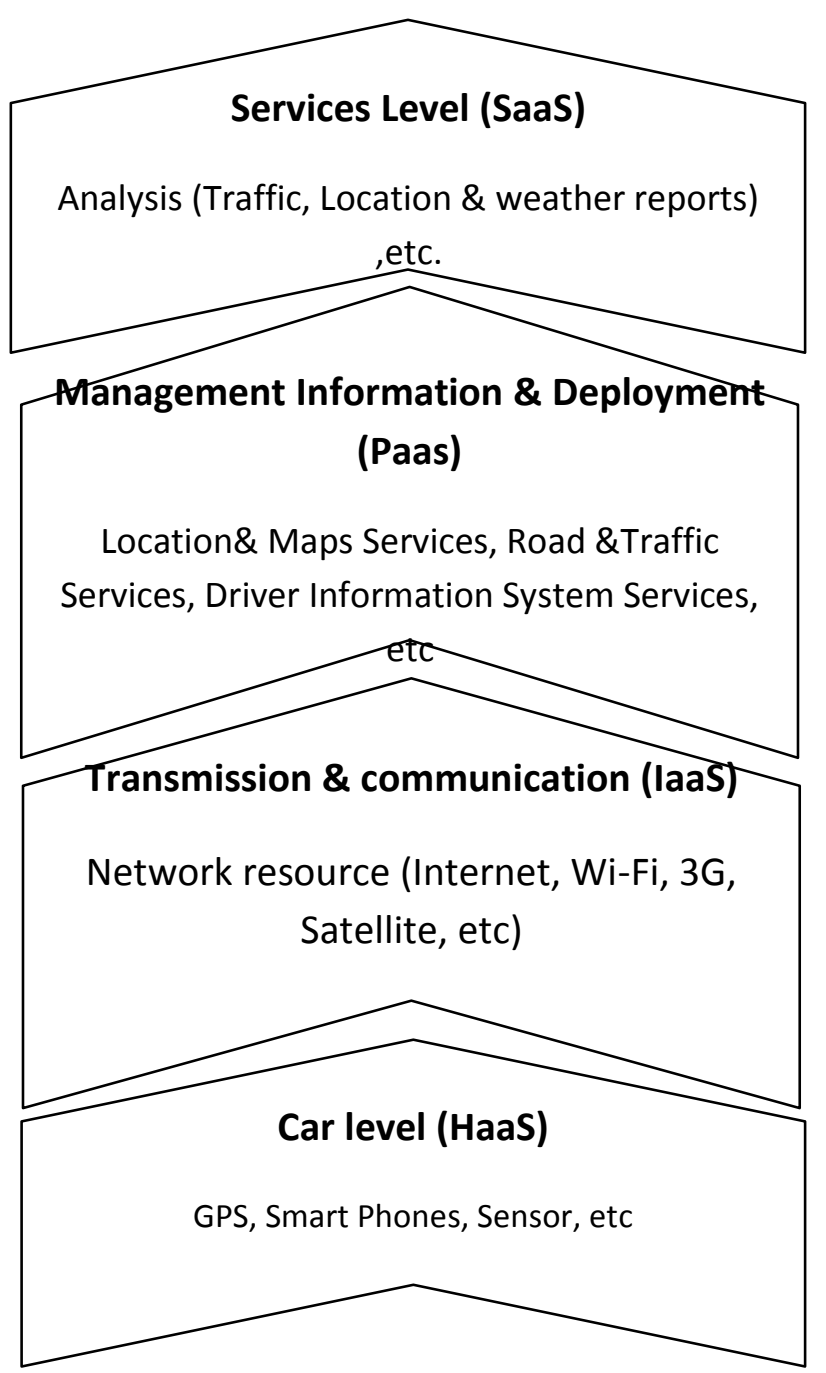

Figure5: The phases of cloud system

4- Services Level: This level is used to integrate vehicle-tovehicle communications and vehicle-to-infrastructure to receive the call and information and then send back the required information like the traffic and road status, location, etc. Data received are analyzed and send back the important decision to increase situational awareness and reduce or eliminate crashes through $\mathrm{V} 2 \mathrm{~V}$ and $\mathrm{V} 2 \mathrm{I}$ data transmission. Besides, it is maximize the driver advisories, driver warnings, and vehicle and/or infrastructure controls. The analysis focused on crash avoidance systems that help drivers in preventing the crashes before it happened. 
The car level will have various devices module ranging such as sensors, WIFI, GSP, smart phone and pc-based devices. It is valuable mentioning system that used to build a body area sensor network (BASN) within a short range. Some of the devices may be prepared with the car whereas the others may be hand-held devices like smart mobile or attached on the driver to monitor the physical health information like sensors for reading pulses, sugar etc. All the communication devices are implemented in the Haas Layer of the cloud.

The wireless communications are classified into three groups: 1 - The satellite communication is implemented using the GPS devices.

2- The wireless networks communication is implemented using smart phone and its component like GSM, 3G and WiFi.

3- Internet communication is implemented using tablet or IPod or any other navigation devices.

The development of car system is improving driver safety and raising their relaxation.

In sake of providing real time measurement and protection services we suggest to deploy the smart devices inside the vehicles or attached to the drivers. The communication of these devices with each other is handled using a body area sensor network (BASN).

On the car level using the sensor layer, we can collect the raw data like temperature, pressure, image and biomedical information and then stored this information in a depository for future processing in the next layer.

The analysis of data will be implemented in the high processing layer based on techniques like data mining, soft computing and reasoning, etc. In conclusion, the outputs of needed information will be delivered through the communicating tools from high layer to the actuator layer to take suitable actions for sending alarm signals to the driver or healthcare centers. The Service Level based the context information will collected and transmits on base of need. This includes the driver status monitoring, Location Maps Services, Road \&Traffic Services, weather report, car itself performance monitoring etc.

The figure No 6 shows the possible architecture of the proposed system which uses cloud services for all the computing needs. The life critical systems need fast and accurate computing services to save life. Our systems can help in providing the required services over the cloud, it will help drivers, passengers, hospitals, insurance companies and traffic police.

\section{CONCLUSION}

Life critical systems like accident handling systems needs lot of services from various fields and an attempt is made in this paper to link the life critical system with new emerging area of cloud computing. Section first introduces the issues of accident handling and various services required for the same. The literature survey of the paper provides the work happened in the area of vehicles and computing and Cloud computing seems to offer some incredible benefits for communicators: the availability of an incredible array of software applications, access to lightning-quick processing power, unlimited storage, and the ability to easily share and process information. All of this is available through browser any time you can access the
Internet. Various services required at vehicles, hospitals and on road side can be provided by the cloud computing solutions.
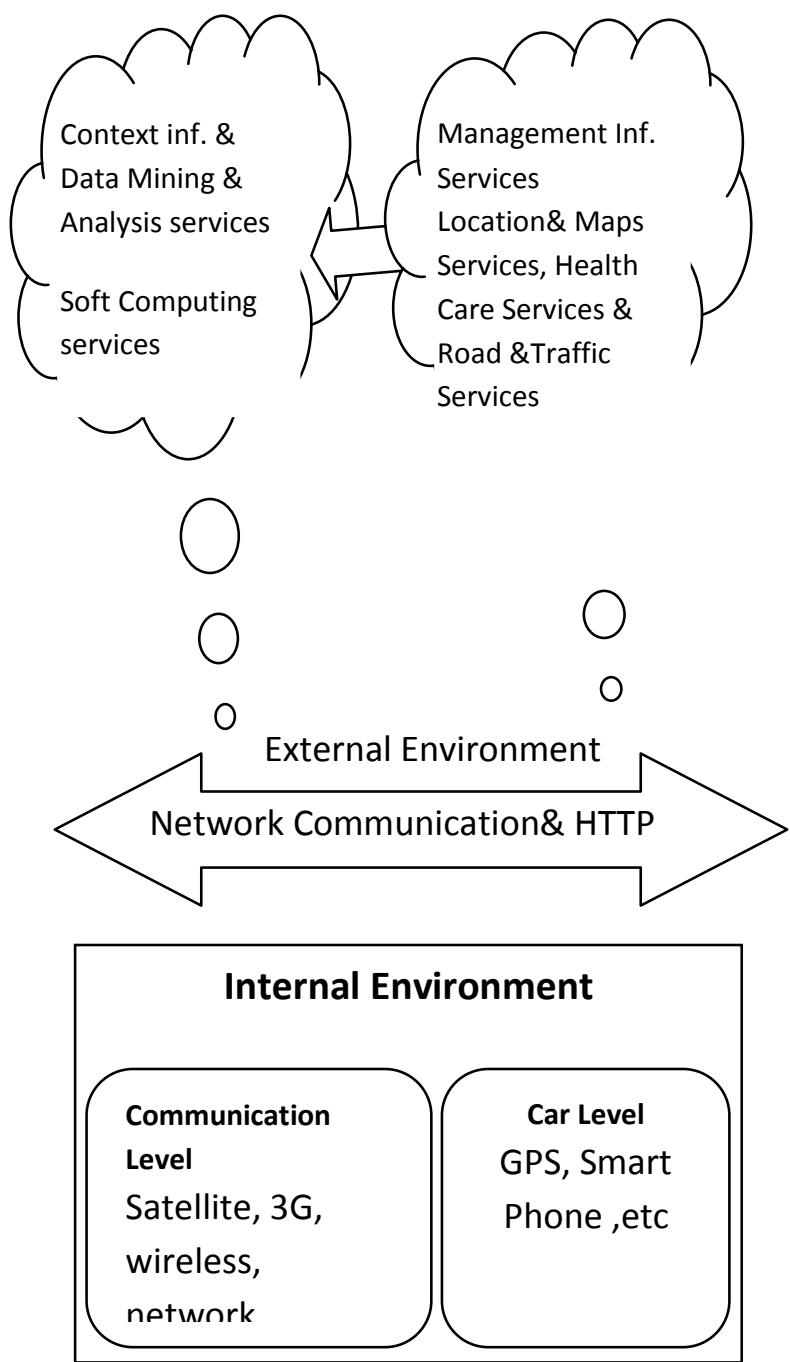

Figure6: The framework of cloud system

\section{REFERENCES}

[1] Brizgalov, Chukhantsev, Fedorkin," Architecture of Traffic Control System Using Cloud Computing", 10 International Conferences and Seminar EDM 2010, July ERLAGOL, pp 215-216.

[2] Dinesh Kumar Saini "Sense the Future" Campus Volume 1- Issue 11, Page No14-17, February 2011.

[3] S L Saini, Dinesh Kumar Saini, and Jabar H. Yousif, "cloud computing and enterprise resource planning systems", Journal of Lecture Notes in Engineering and Computer Science,Vol:2190 ， Issue:1 pp:681-684 , 2011.Issn: 20780958.

[4] Chieu, Mohindar, Karve and Segal, "Dynamic Sacling of Web Applications in a Virtualized Cloud Computing Environment “, IEEE International Conference on EBusiness Engineering, 2009 IEEE ,pp. 281-286

[5] Rodrigo, Ranjan, Anton, Rose, Buyya, "CloudSim: a toolkit for modeling and simulation of cloud computing environments and evaluation of resource provisioning algorithms", June 2010, Jon Wiley and sons. 
[6] Shuai Zhang, Shfen Zhang, Xuebin Chen,Xiuzhen Huo, "Cloud Computing Research and Development Trends", 2010 Second International Conference on Future Networks,2010 IEEE , pp 93-97.

[7] Vecchiola, Pandey, Buyya," High Performance Cloud Computing: A View of Scientific Applications “, 10th International Symposium on Pervasive System, Algorithms and Networks, 2009 IEEE, pp 4-16.

[8] Ron Stence, "Hybrid vehicle control systems" Portable Design, ISSN 1086-1300, Volume 12, Issue 5, p. 28 - 39. 05/2006,

[9] Dinesh Kumar Saini and Jabar H Yousif. Article: Soft Computing Techniques for Mishaps Prediction. International Journal of Computer Applications 47(8):1-7, June 2012. Published by Foundation of Computer Science, New York, USA.

[10] Pearson S (2009) Taking account of privacy when designing cloud computing services. Proceedings of the 2009 ICSE workshop on software engineering challenges of cloud computing, IEEE Comp Soc, pp 44-52

[11] Ristenpart T et al (2009) Hey, you, get off of my cloud: exploring information leakage in third-party compute clouds. Proceeding of ACM conference on computer and communications security

[12] Foster I, Yong Zhao, Raicu I, Lu S (2008) Cloud computing and grid computing 360-degree compared. In: Grid computing environments workshop, 2008. GCE '08grid computing environments workshop, 2008. GCE '08, Auxtin, TX, pp 1-10

[13] Vaquero LM, Rodero-Merino L, Caceres J, Lindner M (2009) A break in the clouds: towards a cloud definition. SIGCOMM Comput Commun Rev 39(1):50-55

[14] Dikaiakos MD, Katsaros D, Mehra P et al (2009) Cloud computing: distributed Internet computing for IT and scientific research. IEEE Internet Comput 5:10-13

[15] Foster I, Zhao Y, Raicu I et al (2008) Cloud computing and grid computing 360-degree compared. In: Proceedings grid computing environments workshop, IEEE Computer Society Press

[16] Miller M. "Cloud computing: web-based applications that change the way you work and collaborate online", Que Publishing, Indianapolis, USA, 2008.
[17] Mather T. "Cloud computing is on the up, but what are the security issues?" Secure Computing Magazine, London march 2009.

[18] ACA Research, Software-as-a-Service (SaaS) in Australia: Is it the Next Big Thing?(2007),http://www.anthonywatson.net.au/files/SA AS\%20White\%20Paper\%20FINAL.PDF (cited March 4, 2008)

[19] Ayala, Daniel; Wolfson, Ouri; Xu, Bo; Dasgupta, Bhaskar; Lin, Jie, "Parking slot assignment games" Proceedings of the 19th ACM SIGSPATIAL International Conference on advances in geographic information systems, 11/2011, GIS '11, ISBN 1450310311 , pp. $299-308,2011$.

[20] R Maggiani. (2009). "Cloud Computing Is Changing How We Communicate". In Proceedings of Professional Communication Conference, 2009, Waikiki, Hawaii. IPCC 2009. IEEE

[21] Nitin B Raut, Jabar H. Yousif,, Sanad Al Maskari, and Dinesh Kumar Saini "cloud for pollution control and global warming" , Journal of Lecture Notes in Engineering and Computer Science,Vol:2190 , Issue:1 pp:681-684, 2011.

[22] Z Shuai, Z Shufen, C Xuebin, H Xiuzhen (2010), “ Cloud Computing Research and Development Trend". In Proceeding of Second International Conference on Future Networks, 2010. ICFN '10. Sanya, Hainan, China

[23] L-J Zhang and Q Zhou (2009),"CCOA: Cloud Computing Open Architecture". In Proceeding of IEEE International Conference on Web Services, 2009. ICWS 2009.

[24] B.P Rimal and E Choi (2009), "A Conceptual Approach for Taxonomical Spectrum of Cloud Computing". In Proceedings of the 4th International Conference on Ubiquitous Information Technologies \& Applications, 2009. ICUT '09. Fukuoka, Japan

[25] Cloud Security Alliance CSA (2010),"Top Threats of Cloud Computing V1.0", Retrieved 04 04, 2010, from http://www.cloudsecurityalliance.org/topthreats 